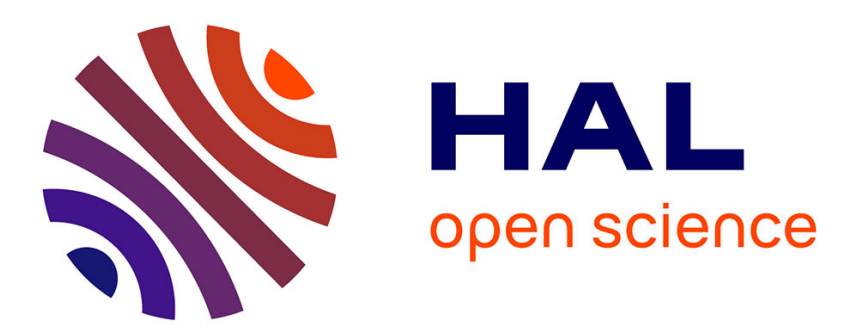

\title{
Helmholtz decomposition of vector fields with mixed boundary conditions and an application to a posteriori finite element error analysis of the Maxwell system
}

Emmanuel Creusé, Serge Nicaise, Zuqi Tang

\section{- To cite this version:}

Emmanuel Creusé, Serge Nicaise, Zuqi Tang. Helmholtz decomposition of vector fields with mixed boundary conditions and an application to a posteriori finite element error analysis of the Maxwell system. Mathematical Methods in the Applied Sciences, 2015, 38 (4), pp.738-750. 10.1002/mma.3104 . hal-00777690

\author{
HAL Id: hal-00777690 \\ https://hal.science/hal-00777690
}

Submitted on 17 Jan 2013

HAL is a multi-disciplinary open access archive for the deposit and dissemination of scientific research documents, whether they are published or not. The documents may come from teaching and research institutions in France or abroad, or from public or private research centers.
L'archive ouverte pluridisciplinaire $\mathbf{H A L}$, est destinée au dépôt et à la diffusion de documents scientifiques de niveau recherche, publiés ou non, émanant des établissements d'enseignement et de recherche français ou étrangers, des laboratoires publics ou privés. 


\title{
Helmholtz decomposition of vector fields with mixed boundary conditions and an application to a posteriori finite element error analysis of the Maxwell system
}

\author{
Emmanuel Creusé, Serge Nicaiseł Zuqi Tang ${ }^{\ddagger}$
}

July 18, 2012

\begin{abstract}
This paper is devoted to the derivation of a Helmholtz decomposition of vector fields in the case of mixed boundary conditions imposed on the boundary of the domain. This particular decomposition allows to obtain a residual a posteriori error estimator for the approximation of magnetostatic problems given in the so-called $A$ formulation, for which the reliability can be established. Numerical tests confirm the obtained theoretical predictions.
\end{abstract}

AMS (MOS) subject classification 35Q61; 65N30; 65N15; 65N50.

Key Words Helmholtz decomposition, Finite element methods, A posteriori error estimators.

\section{Introduction}

Nowadays, the finite element method is widely used to study electromagnetic systems. In order to carry out an adaptive mesh refinement strategy as well as to control the error obtained between the numerical solution and the exact one, local a posteriori error estimators are needed. Since the pioneering work due to Babuska and Rheinboldt [3, 4], and then several monographies related to this subject $[1,5,26]$, an impressive number of papers have been published, considering several kinds of equations, numerical methods or approximation spaces, and providing a large variety of error estimators. In this work, we are particularly interested in explicit residual-based error estimators, devoted to the

${ }^{*}$ LPP, Université Lille 1, Cité Scientifique, 59655 Villeveuve d'Ascq Cedex \& EPI SIMPAF, INRIA Lille Nord Europe, France. creuse@math.univ-lille1.fr.

${ }^{\dagger}$ LAMAV, Université de Valenciennes et du Hainaut Cambrésis, FR CNRS 2956, Institut des Sciences et Techniques de Valenciennes, F-59313 - Valenciennes Cedex 9 France (corresponding author).

${ }^{\ddagger}$ L2EP, Université Lille 1, Cité Scientifique, 59655 Villeveuve d’Ascq Cedex, France. 
approximation of the solutions of Maxwell equations, discretized by Nédélec $H$ (curl) conforming edge elements.

For the last twenty years, these error estimators have been developped for the computation of quasistatic electromagnetic fields in conductors in the case of regular data and domains [6]. They have been then generalized to the case of anisotropic meshes and non regular data [21], as well as to the one of Lipschitz domains [25]. The robustness of the estimations with the data was also addressed in [11]. More recently, in the same kind of work, namely explicit residual-based a posteriori estimators have also been developped for quasistatic magnetodynamic problems, modeled with harmonic potential formulations $[9,13,27,28]$. Moreover, some papers are now devoted to the way to design some adaptive mesh loop strategies, in order to ensure the convergence process while the mesh is being refined using the error estimators [10, 17, 18].

In most of the above quoted papers, the reliability of the estimator (namely, the fact that the error is bounded from above by the estimator) is based on a suitable orthogonal Helmholtz decomposition of the error. This one is needed to allow the use of some suitable interpolation operators, which are crucial in the proofs. Nevertheless, to our best knowledge, the case corresponding to mixed boundary conditions imposed on the vector field (see the boundary conditions on $A$ arising in (3) below) have not been reported yet. Consequently, the main objective of this paper is to prove the corresponding Helmholtz decomposition, in order to extend the reliability of residual-type estimator to this case.

The schedule of the paper is the following. In section 1, the context of the problem is recalled. The continuous model we are interested in is given, the existence and uniqueness of the solution are established, and the finite element approximation is briefly described. Section 2 constitutes the main part of this work and is devoted to the Helmholtz decomposition of vector fields. Several lemmas are proved, and used to derive Theorem 2.3. Consequently, in section 3 we are able to derive the a posteriori residual error estimator. With the previous Helmholtz decomposition in hands, the proof can be easily derived from previous papers, but we chose to recall its main steps to make this work self-contained. Finally, section 4 is devoted to numerical tests, to underline the good correspondence between theoretical and numerical results obtained.

\section{Setting of the problem}

\subsection{Modeling and continuous variational formulation}

Let us consider an open simply connected domain $\Omega \subset \mathbb{R}^{3}$ with a Lipschitz boundary $\Gamma=\partial \Omega$. In this paper, we are interested in the magnetic flux density $B$ induced in $\Omega$ by 
an eddy current $J_{s}$, modeled by the following stationary Maxwell equations :

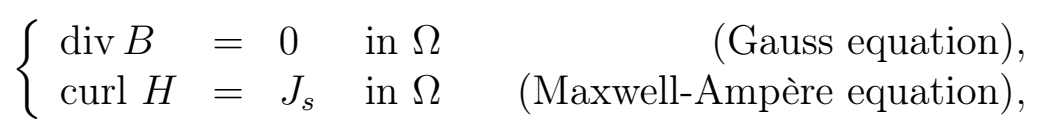

where $H$ is the magnetic field defined by $B=\mu H$, with $\mu \in L^{\infty}(\Omega)$ the magnetic permeability of the medium, for which we assume that there exists $\mu_{\min } \in \mathbb{R}_{+}^{*}$ such that $\mu \geq \mu_{\min }$ in $\Omega$. Let us moreover suppose that $\Gamma=\Gamma_{N} \cup \Gamma_{T}$, with $\Gamma_{N} \cap \Gamma_{T}=\emptyset$ and $\Gamma_{N}$ connected. Finally we denote by $n$ the unit outward normal to $\Omega$ on $\Gamma$. Usual boundary conditions on $B$ associated with problem (1) are given by :

$$
\left\{\begin{array}{l}
B \cdot n=0 \quad \text { on } \Gamma_{N}, \\
\frac{1}{\mu} B \times n=0
\end{array}\right.
$$

Then, a vector potential $A$ is introduced such that $B=\operatorname{curl} A$, and problem (1)-(2) is reformulated in its potential formulation given by :

$$
\left\{\begin{aligned}
\operatorname{curl}\left(\frac{1}{\mu} \operatorname{curl} A\right) & =J_{s} & & \text { in } \Omega, \\
A \times n & =0 & & \text { on } \Gamma_{N}, \\
A \cdot n=0 \text { and } \frac{1}{\mu} \operatorname{curl} A \times n & =0 & & \text { on } \Gamma_{T}, \\
\operatorname{div} A & =0 & & \text { in } \Omega .
\end{aligned}\right.
$$

Let us note that the divergence-free constraint on $A$ corresponds to the gauge condition, needed to ensure the uniqueness of the solution. Here, $J_{s}$ is supposed to be given such that :

$$
\left\{\begin{array}{rll}
\operatorname{div} J_{s}= & 0 & \text { in } \Omega \\
J_{s} \cdot n= & & \text { on } \Gamma_{T} .
\end{array}\right.
$$

In the following, for a given domain $D$, the $L^{2}(D)$ (or $\left(L^{2}(D)\right)^{3}$ ) norm will be denoted by $\|\cdot\|_{D}$. The usual norm and semi-norm of $H^{1}(D)\left(\right.$ or $\left.\left(H^{1}(D)\right)^{3}\right)$ will be denoted $\|\cdot\|_{1, D}$ and $|\cdot|_{1, D}$ respectively. We first introduce the following functional spaces:

$$
\begin{gathered}
X(\Omega)=\left\{A \in L^{2}(\Omega)^{3}: \operatorname{curl} A \in L^{2}(\Omega)^{3} \text { and } A \times n=0 \text { on } \Gamma_{N}\right\}, \\
H_{\Gamma_{N}}^{1}(\Omega)=\left\{\varphi \in H^{1}(\Omega): \varphi=0 \text { on } \Gamma_{N}\right\}, \\
X^{0}(\Omega)=\left\{A \in X(\Omega): \int_{\Omega} A \cdot \nabla \varphi=0 \forall \varphi \in H_{\Gamma_{N}}^{1}(\Omega)\right\},
\end{gathered}
$$

with the natural norm $\|\cdot\|_{X(\Omega)}$ defined on $X(\Omega)$ by :

$$
\|A\|_{X(\Omega)}^{2}=\|A\|_{\Omega}^{2}+\|\operatorname{curl} A\|_{\Omega}^{2}
$$


Then, we consider the variational formulation associated with (3) by looking for $A \in X^{0}(\Omega)$ such that, for all $A^{\prime} \in X^{0}(\Omega)$, we have :

$$
a\left(A, A^{\prime}\right)=l\left(A^{\prime}\right),
$$

with :

$$
a\left(A, A^{\prime}\right)=\int_{\Omega} \frac{1}{\mu} \operatorname{curl} A \cdot \operatorname{curl} A^{\prime} \quad \text { and } \quad l\left(A^{\prime}\right)=\int_{\Omega} J_{s} \cdot A^{\prime} .
$$

In order to address the question of the well-posedness of the problem, let us introduce the space $X_{M}(\Omega)$ defined by :

$X_{M}(\Omega)=\left\{A \in L^{2}(\Omega)^{3}: \operatorname{curl} A \in L^{2}(\Omega)^{3}, \operatorname{div} A \in L^{2}(\Omega), A \times n=0\right.$ on $\Gamma_{N}$ and $A \cdot n=0$ on $\left.\Gamma_{T}\right\}$, with its associated natural norm $\|\cdot\|_{X_{M}(\Omega)}$ defined by :

$$
\|A\|_{X_{M}(\Omega)}^{2}=\|A\|_{\Omega}^{2}+\|\operatorname{div} A\|_{\Omega}^{2}+\|\operatorname{curl} A\|_{\Omega}^{2}
$$

Since $X_{M}(\Omega)$ is compactly embedded in $L^{2}(\Omega)^{3}[24]$, and since it can be moreover easily shown that $X^{0}(\Omega) \subset X_{M}(\Omega)$, the following Friedrichs-type inequality holds :

There exists $C>0$ such that for all $A \in X^{0}(\Omega)$, we have :

$$
\|A\|_{\Omega} \leq C\|\operatorname{curl} A\|_{\Omega}
$$

Consequently, we deduce from the Lax-Milgram lemma that problem (5) is well-posed and admits a unique solution $A \in X^{0}(\Omega)$.

Now, let us derive the following property :

Lemma 1.1 Let $A \in X^{0}(\Omega)$ be the unique solution of (5). Then for all $B \in X(\Omega)$, we have

$$
a(A, B)=l(B) .
$$

Proof. Since $B \in X(\Omega)$, we can decompose it using the following Helmholtz decomposition:

$$
B=\Psi+\nabla \tau,
$$

with $\Psi \in X^{0}(\Omega)$ and $\tau \in H_{\Gamma_{N}}^{1}(\Omega)$. Indeed, $\tau \in H_{\Gamma_{N}}^{1}(\Omega)$ is simply defined by

$$
\int_{\Omega} \nabla \tau \cdot \nabla \varphi=\int_{\Omega} B \cdot \nabla \varphi, \forall \varphi \in H_{\Gamma_{N}}^{1}(\Omega),
$$

and $\Psi=B-\nabla \tau$. So we may write

$$
a(A, B)=a(A, \Psi)=l(\Psi)
$$

Because of (4) and the fact that $\tau_{\mid \Gamma_{N}}=0$, we clearly have $l(\Psi)=l(B)$, so that $(7)$ holds. 


\subsection{Finite Element approximation}

Problem (5) is approximated using a finite element method. The domain $\Omega$ is from now supposed to be polyhedral, and is discretized by a conforming mesh $\mathscr{T}_{h}$ made of tetrahedra denoted by $T$. The magnetic permeability of the medium $\mu$ is supposed to be constant over each tetrahedron. We denote by $h_{T}$ the diameter of $T$ and by $\rho_{T}$ the diameter of its largest inscribed ball. For any element $T$, the ratio $h_{T} / \rho_{T}$ is supposed to be bounded from above by a constant $\alpha>0$ independant of $T$ and of the mesh size $h=\max _{T \in \mathscr{T}_{h}} h_{T}$. The faces of $\mathscr{T}_{h}$ are denoted by $F$, and we denote by $h_{F}$ the diameter of the face $F$. For compatibility reasons, we suppose that only one boundary condition can be prescribed on a given boundary face $F \subset \Gamma$. In other words, if $F \subset \Gamma$, we assume that either $F \subset \Gamma_{N}$ or $F \subset \Gamma_{T}$. Finally, the set of the faces of the triangulation is denoted by $\mathcal{F}$, whereas the set of the internal faces to $\Omega$ is denoted by $\mathcal{F}_{\text {int }}$.

The approximation spaces are now defined by :

$$
\begin{gathered}
X_{h}(\Omega)=X(\Omega) \cap \mathcal{N D}_{1}\left(\Omega, \mathscr{T}_{h}\right)=\left\{A_{h} \in X(\Omega): A_{h \mid T} \in \mathcal{N D}_{1}(T), \forall T \in \mathscr{T}_{h}\right\}, \\
\mathcal{N D}_{1}(T)=\left\{A_{h}: \begin{array}{l}
T \longrightarrow \\
x \longrightarrow a+b \times x
\end{array}, a, b \in \mathbb{R}^{3}\right\}, \\
\Theta_{h}^{0}(\Omega)=\left\{\xi_{h} \in H_{\Gamma_{N}}^{1}(\Omega): \xi_{h \mid T} \in \mathbb{P}_{1}(T) \forall T \in \mathscr{T}_{h}\right\}, \\
X_{h}^{0}(\Omega)=\left\{A_{h} \in X_{h}(\Omega): \int_{\Omega} A_{h} \cdot \nabla \xi_{h}=0 \forall \xi_{h} \in \Theta_{h}^{0}(\Omega)\right\} .
\end{gathered}
$$

The discrete variational formulation now consists in finding $A_{h} \in X_{h}^{0}(\Omega)$ such that, for all $A^{\prime} \in X_{h}^{0}(\Omega)$, we have :

$$
a\left(A_{h}, A_{h}^{\prime}\right)=l\left(A_{h}^{\prime}\right) .
$$

Because of this finite element choice, and from a discrete compactness property of $X_{h}^{0}(\Omega)$ $[19,22]$, we have, as for the continuous case, that the discrete problem (8) is well-posed and admits a unique solution $A_{h} \in X_{h}^{0}(\Omega)$. Let us remark that the approximation is sought on the Nédélec functions that are discrete divergence-free. Nevertheless, in practice, the corresponding gauge condition is not explicitly forced. Indeed, thanks to the particular iterative solver used for the resolution of the linear system, the divergence-free property of the successive vector fields obtained is preserved, so that the gauge condition is implicitly imposed [23].

\section{Helmholtz decomposition of vector fields}

The aim of this section is to obtain Theorem 2.3, which will be used in section 3 to derive the a posteriori error estimate. 
To begin with, for $v \in X_{M}(\Omega)$, we remark that curl $v$ belongs to

$$
\hat{J}(\Omega):=\left\{v \in L^{2}(\Omega)^{3}: \operatorname{div} v=0 \text { and } v \cdot n=0 \text { on } \Gamma_{N}\right\} .
$$

According to Lemmas 3.1 and 3.2 of [12] we first consider smooth vector potentials for elements of $\hat{J}(\Omega)$. For that purpose we combine some ideas from Theorems 3.1 and 4.1 of [7] (see also [8]). First we state a technical result.

Lemma 2.1 Let $A$ be a bounded domain of $\mathbb{R}^{3}$ with a Lipschitz boundary. Then there exists a linear continuous operator $E_{A}$ from $H^{1}(A)$ into $H_{\mathrm{loc}}^{1}\left(\mathbb{R}^{3}\right)$ such that

$$
E_{A} 1=1,
$$

and fulfilling the usual properties of an extension operator:

$$
E_{A} u=u \text { on } A, \forall u \in H^{1}(A),
$$

and for all open sets $B$ of $\mathbb{R}^{3}$ containing $\bar{A}$, there exists a positive constant $C$ depending only on $A$ and $B$ such that

$$
\left\|E_{A} u\right\|_{1, B} \leq C\|u\|_{1, A}, \forall u \in H^{1}(A) .
$$

Proof. By Theorem 1.4.3.1 of [16], there exists a linear continuous operator $E$ from $H^{1}(A)$ into $H^{1}\left(\mathbb{R}^{3}\right)$ such that

$$
E u=u \text { on } A, \forall u \in H^{1}(A),
$$

and

$$
\|E u\|_{1, \mathbb{R}^{3}} \leq C\|u\|_{1, A}, \forall u \in H^{1}(A),
$$

for some constant $C_{1}>0$. An appropriate choice for $E_{A}$ is

$$
E_{A} u=E\left(u-\mathcal{M}_{A} u\right)+\mathcal{M}_{A} u, \forall u \in H^{1}(A),
$$

where $\mathcal{M}_{A} u$ is the mean of $u$ in $A$, namely

$$
\mathcal{M}_{A} u=|A|^{-1} \int_{A} u(x) d x
$$

when $|A|$ is the mesure of $A$. The definition of $E_{A}$ and the fact that $E$ is linear imply that (9) holds. In the same manner we see that (10) is valid because

$$
E\left(u-\mathcal{M}_{A} u\right)=u-\mathcal{M}_{A} u \text { on } A .
$$

Finally the continuity property of $E_{A}$ comes from the continuity of $E$ and the fact that

$$
\left\|\mathcal{M}_{A} u\right\|_{1, B}=\left\|\mathcal{M}_{A} u\right\|_{B}=\frac{|B|^{1 / 2}}{|A|^{1 / 2}}\left\|\mathcal{M}_{A} u\right\|_{A} \leq \frac{|B|^{1 / 2}}{|A|^{1 / 2}}\|u\|_{A} .
$$


Lemma 2.2 For all $f \in \hat{J}(\Omega)$ there exists $w \in H^{1}(\Omega)^{3}$ such that

$$
w \times n=0 \text { on } \Gamma_{N}
$$

and fulfilling

$$
\operatorname{curl} w=f \text { in } \Omega
$$

and

$$
\|w\|_{1, \Omega} \leq C\|f\|_{\Omega},
$$

for some constant $C>0$ depending only on $\Omega$.

Proof. As said before we combine some arguments from Theorem 3.1 of [7] (case $\Gamma_{T}=\emptyset$ ) and from Theorem 4.1 of [7] ( $\left.\operatorname{case} \Gamma_{N}=\emptyset\right)$.

Step 1 We consider $\tilde{\Omega}$ a bounded domain of $\mathbb{R}^{3}$ with a polyhedral boundary such that

$$
\Omega \subset \tilde{\Omega},
$$

and such that the common faces between $A:=\tilde{\Omega} \backslash \Omega$ and $\Omega$ are the faces of $\Gamma_{T}$. As $A$ enters in the framework of Lemma 2.1, we can use the extension operator $E_{A}$.

Step 2 Set

$$
H_{m}^{1}(A):=\left\{u \in H^{1}(A): \int_{A} u(x) d x=0\right\},
$$

that can be equipped with the $H^{1}$-semi norm. Let $u \in H_{m}^{1}(A)$ be the unique solution of

$$
\int_{A} \nabla u \cdot \nabla w d x=\int_{\Omega} f \cdot \nabla E_{A} w d x, \forall w \in H_{m}^{1}(A) .
$$

This solution exists and is unique owing to the Lax-Milgram lemma because the right-hand side is a linear and continuous form on $H_{m}^{1}(A)$. Furthermore as $E_{A} 1=1,(14)$ is valid on the whole of $H^{1}(A)$, namely we also have

$$
\int_{A} \nabla u \cdot \nabla w d x=\int_{\Omega} f \cdot \nabla E_{A} w d x, \forall w \in H^{1}(A) .
$$

Now we set

$$
\tilde{f}:= \begin{cases}f & \text { in } \Omega, \\ -\nabla u & \text { in } A\end{cases}
$$

Let us check that $\tilde{f}$ belongs to

$$
J(\nu, \tilde{\Omega}):=\left\{v \in L^{2}(\Omega)^{3}: \operatorname{div} v=0 \text { and } v \cdot n=0 \text { on } \partial \tilde{\Omega}\right\} .
$$

Since it is well known that $\tilde{f} \in J(\nu, \tilde{\Omega})$ if and only if

$$
\int_{\tilde{\Omega}} \tilde{f} \cdot \nabla h d x=0, \forall h \in H^{1}(\tilde{\Omega}),
$$


it remains to check this last property. For an arbitrary $h \in H^{1}(\tilde{\Omega})$, by the definition of $\tilde{f}$, we have

$$
\int_{\tilde{\Omega}} \tilde{f} \cdot \nabla h d x=\int_{\Omega} f \cdot \nabla h d x-\int_{A} \nabla u \cdot \nabla h d x .
$$

Since $u$ satisfies (15) we get

$$
\int_{\tilde{\Omega}} \tilde{f} \cdot \nabla h d x=\int_{\Omega} f \cdot \nabla h d x-\int_{\Omega} f \cdot \nabla E_{A} h d x=\int_{\Omega} f \cdot \nabla\left(h-E_{A} h\right) d x .
$$

Hence by Green's formula in $\Omega$ we obtain

$$
\int_{\tilde{\Omega}} \tilde{f} \cdot \nabla h d x=-\int_{\Omega} \operatorname{div} f\left(h-E_{A} h\right) d x+\left\langle f \cdot n ; h-E_{A} h\right\rangle_{\left(\tilde{H}^{1 / 2}\left(\Gamma_{N}\right)\right)^{\prime}-\tilde{H}^{1 / 2}\left(\Gamma_{N}\right)},
$$

reminding that $h=E_{A} h$ on $\Gamma_{T}$. Since $f$ belongs to $\hat{J}(\Omega)$, this last right-hand side is zero and we have proven (16).

Step 3 Fix a ball $B$ of $\mathbb{R}^{3}$ such that

$$
\overline{\widetilde{\Omega}} \subset B,
$$

and set

$$
g:= \begin{cases}\tilde{f} & \text { in } \tilde{\Omega} \\ 0 & \text { in } B \backslash \tilde{\Omega} .\end{cases}
$$

As $\tilde{f} \in J(\nu, \tilde{\Omega}), g$ belongs to $J(\nu, B)$. Therefore by [7], there exists $w_{0} \in H^{1}(B)^{3}$ such that

$$
\operatorname{curl} w_{0}=g \text { and } \operatorname{div} w_{0}=0 \text { in } B
$$

with

$$
\left\|w_{0}\right\|_{1, B} \leq C_{1}\|g\|_{B}=C_{1}\|\tilde{f}\|_{\tilde{\Omega}}
$$

where $C_{1}$ is a positive constant that depends only on $B$. But according to the definition of $\tilde{f}$

$$
\|\tilde{f}\|_{\tilde{\Omega}}^{2}=\|f\|_{\Omega}^{2}+\|\nabla u\|_{A}^{2},
$$

and by (14), Cauchy-Schwarz's inequality and Lemma 2.1 we get

$$
\|\nabla u\|_{A} \leq C_{2}\|f\|_{\Omega}
$$

where $C_{2}$ is a positive constant that depends only on $A$ and $B$. Therefore the estimate (18) reduces to

$$
\left\|w_{0}\right\|_{1, B} \leq C_{3}\|f\|_{\Omega} .
$$

where $C_{3}$ is a positive constant that depends only on $A$ and $B$.

Step 4 As $g=0$ on $B \backslash \tilde{\Omega}$, we get

$$
\operatorname{curl} w_{0}=0 \text { in } B \backslash \tilde{\Omega} .
$$


Since this set is simply connected, there exists $\Phi_{A} \in H^{1}(B \backslash \tilde{\Omega})$ such that

$$
w_{0}=\nabla \Phi_{A} \text { in } B \backslash \tilde{\Omega} .
$$

This function $\Phi_{A}$ even belongs to $H^{2}(B \backslash \tilde{\Omega})$ since $w_{0} \in H^{1}(B)^{3}$.

Denote by $\Pi$ the extension (linear continuous) operator from $H^{2}(B \backslash \tilde{\Omega})$ into $H^{2}(B)$ such that

$$
\Pi w=w \text { in } B \backslash \tilde{\Omega} .
$$

Now setting

$$
\Phi=\Pi \Phi_{A}
$$

we notice that

$$
w=w_{0}-\nabla \Phi
$$

yields the conclusion. Indeed $w$ belongs to $H^{1}(\Omega)^{3}$ by the regularity of $w_{0}$ and $\Phi$. Furthermore

$$
\operatorname{curl} w=\operatorname{curl} w_{0}
$$

and therefore by (17), we obtain (12) (as $g=f$ in $\Omega$ ). Finally

$$
w \times n=w_{0} \times n-\nabla \Phi \times n \text { on } \Gamma_{N} .
$$

and by (20),

$$
w \times n=\nabla \Phi_{A} \times n-\nabla \Pi \Phi_{A} \times n=0 \text { on } \Gamma_{N} .
$$

by the property of the extension operator and proves (11).

Theorem 2.3 If $\Omega$ is simply connected and $\Gamma_{N}$ is connected, then for any $u \in X_{M}(\Omega)$ there exist $w \in H^{1}(\Omega)^{3}$ satisfying (11) and $\varphi \in H_{\Gamma_{N}}^{1}(\Omega):=\left\{\psi \in H^{1}(\Omega): \psi=0\right.$ on $\left.\Gamma_{N}\right\}$ satisfying $\operatorname{div} \nabla \varphi \in L^{2}(\Omega)$ and such that the following Helmholtz decomposition

$$
u=w+\nabla \varphi \text { in } \Omega
$$

holds with the estimate

$$
\|w\|_{1, \Omega}+\|\varphi\|_{1, \Omega}+\|\operatorname{div} \nabla \varphi\|_{\Omega} \leq C\|u\|_{X_{M}(\Omega)},
$$

for some constant $C>0$ depending only on $\Omega$.

Proof. For $u \in X_{M}(\Omega)$ consider $v=\operatorname{curl} u$ that belongs to $\hat{J}(\Omega)$. Hence by Lemma 2.2, there exists $w \in H^{1}(\Omega)^{3}$ satisfying (11) and fulfilling

$$
\operatorname{curl} w=\operatorname{curl} u \text { in } \Omega \text {, }
$$

and

$$
\|w\|_{1, \Omega} \leq C\|\operatorname{curl} u\|_{\Omega}
$$


for some constant $C>0$ depending only on $\Omega$. This means that $u-w$ is curl free and since $\Omega$ is simply connected, there exists $\varphi_{0} \in H^{1}(\Omega)$ such that

$$
u-w=\nabla \varphi_{0} \text { in } \Omega .
$$

As $(u-w) \times n=0$ on $\Gamma_{N}$, we deduce that

$$
\nabla \varphi_{0} \times n=0 \text { on } \Gamma_{N} .
$$

Consequently there exists a constant $K \in \mathbb{C}$ such that (reminding that $\Gamma_{N}$ is connected)

$$
\varphi_{0}=K \text { on } \Gamma_{N}
$$

The conclusion follows by taking

$$
\varphi=\varphi_{0}-K
$$

since $\nabla \varphi=\nabla \varphi_{0}$ and using (23) and (24).

Remark 2.4 The previous result still holds if $\Gamma_{N}$ is not connected, but in that case $\varphi$ will be constant on each connected component of $\Gamma_{N}$. Similarly the previous result also holds if $\Omega$ is not simply connected, indeed it suffices to apply the previous theorem to each connected component of $\Omega$.

With additional low geometrical assumptions, it is possible to recover homogeneous Neumann boundary conditions for $\varphi$ involved in the Helmholtz decomposition, this is the subject of Theorem 2.5. Nevertheless, it will not be useful in section 3 to derive the a posterior error analysis.

Theorem 2.5 Assume that $\Omega$ is simply connected and $\Gamma_{N}$ is connected. Assume furthermore that

i) for all edges e in common between a face of $\Gamma_{T}$ and a face of $\Gamma_{N}$, the interior angle $\omega_{e}$ is different from $\pi / 2$,

ii) for all corners $c$, the eigenvalues $\lambda_{c, k}^{\text {mixed }}$ of $\Delta_{c}^{\text {mixed }}$ are different from $3 / 4$, where $\Delta_{c}^{\text {mixed }}$ is the non negative Laplace-Beltrami on the unit sphere with mixed boundary conditions (in accordance with the partition of the faces of $\Gamma_{N}$ (Dirichlet) and of $\Gamma_{T}$ (Neumann) containing c).

Then for any $u \in X_{M}(\Omega)$ there exist $w_{0} \in H^{1}(\Omega)^{3} \cap X_{M}(\Omega)$ and $\varphi_{0} \in H_{\Gamma_{N}}^{1}(\Omega)$ satisfying $\operatorname{div} \nabla \varphi_{0} \in L^{2}(\Omega)$ and

$$
\frac{\partial \varphi_{0}}{\partial n}=0 \text { on } \Gamma_{T}
$$

and such that the following Helmholtz decomposition

$$
u=w_{0}+\nabla \varphi_{0} \text { in } \Omega
$$

holds with the estimate

$$
\left\|w_{0}\right\|_{1, \Omega}+\left\|\varphi_{0}\right\|_{1, \Omega}+\left\|\operatorname{div} \nabla \varphi_{0}\right\|_{\Omega} \leq C\|u\|_{X_{M}(\Omega)},
$$

for some constant $C>0$ depending only on $\Omega$. 
Proof. In fact the previous Theorem shows that $\varphi \in H_{\Gamma_{N}}^{1}(\Omega)$ a weak solution of the boundary value problem

$$
\begin{cases}\operatorname{div} \nabla \varphi=\operatorname{div} u-\operatorname{div} w & \text { in } \Omega \\ \frac{\partial \varphi}{\partial n}=-w \cdot n & \text { on } \Gamma_{T}\end{cases}
$$

Since the assumptions on $u$ and the regularity of $w$ garantee that div $u$ - div $w$ belongs to $L^{2}(\Omega)$ and since $w \cdot n$ belongs to $H^{1 / 2}\left(\Gamma_{T}\right)$, we can apply the results from [14] to obtain the next decomposition of $\varphi$ :

$$
\varphi=\varphi_{R}+\varphi_{0}
$$

where $\varphi_{R} \in H^{2}(\Omega)$ and $\varphi_{0} \in H_{\Gamma_{N}}^{1}(\Omega)$ such that

$$
\left\{\begin{array}{l}
\operatorname{div} \nabla \varphi_{0} \in L^{2}(\Omega) \\
\frac{\partial \varphi}{\partial n}=0 \text { on } \Gamma_{T} .
\end{array}\right.
$$

with the estimate

$$
\left\|\varphi_{R}\right\|_{2, \Omega}+\left\|\varphi_{0}\right\|_{1, \Omega}+\left\|\operatorname{div} \nabla \varphi_{0}\right\|_{\Omega} \leq c\left(\|\operatorname{div} u-\operatorname{div} w\|_{\Omega}+\|w \cdot n\|_{H^{1 / 2}\left(\Gamma_{T}\right)}\right),
$$

where $c$ is a positive constant depending only on $\Omega$. We then conclude by taking

$$
w_{0}=w+\nabla \varphi_{R},
$$

where $w \in H^{1}(\Omega)^{3}$ is the vector potential from Theorem 2.3.

\section{A posteriori residual error analysis}

Let us define $\pi_{h}$ as the projection operator from $H(\operatorname{div}, \Omega)=\left\{u \in L^{2}(\Omega)^{3} ; \operatorname{div} u \in L^{2}(\Omega)\right\}$ to an ad hoc discrete approximation space (which can be chosen for example as the low order Raviart-Thomas finite element space [15] so that it is included in $H(\operatorname{div}, \Omega)$ ), and let us denote by $[u]_{F}$ the jump of the quantity $u$ over a face $F$ of the mesh. For each $T \in \mathscr{T}_{h}$, the local error indicator $\eta_{T}$ is defined by :

$$
\eta_{T}^{2}=\eta_{T, 1}^{2}+\eta_{T, 2}^{2}+\sum_{F \subset \partial T, F \in \mathcal{F}_{\text {int }}} \eta_{F, 1}^{2}+\sum_{F \subset \partial T, F \subset \Gamma_{T}} \eta_{F, 2}^{2},
$$

with :

$$
\begin{aligned}
\eta_{T, 1} & =h_{T}\left\|\pi_{h} J_{s}-\operatorname{curl}\left(\frac{1}{\mu} \operatorname{curl} A_{h}\right)\right\|_{T}, \\
\eta_{T, 2} & =h_{T}\left\|J_{s}-\pi_{h} J_{s}\right\|_{T}, \\
\eta_{F, 1} & =h_{F}^{1 / 2}\left\|\left[\frac{1}{\mu} \operatorname{curl} A_{h} \times n\right]_{F}\right\|_{F}, \\
\eta_{F, 2} & =h_{F}^{1 / 2}\left\|\frac{1}{\mu} \operatorname{curl} A_{h} \times n\right\|_{F} .
\end{aligned}
$$


Then, the global error indicator $\eta$ is defined by :

$$
\eta^{2}=\sum_{T \in \mathscr{T}_{h}} \eta_{T}^{2}
$$

Theorem 3.1 Let us suppose that $\Omega$ is simply connected and $\Gamma_{N}$ is connected, there exists a constant $C_{u p}>0$, which does not depend on $A, A_{h}$ or $h$, such that:

$$
\left(\int_{\Omega} \frac{1}{\mu}\left|\operatorname{curl}\left(A-A_{h}\right)\right|^{2}\right)^{\frac{1}{2}} \leq C_{u p} \eta .
$$

Proof. The proof can be easily derived from previous more general works (for example [13] which deals with the magnetodynamic case), but in the restricted case $\Gamma_{T}=\emptyset$ (or, equivalently, $\Gamma_{N}=\Gamma$ ). The novelty of this result is the generalization to the case $\Gamma_{T} \neq \emptyset$, thanks to the new Helmholtz decomposition (21) and to (22). The sketch of the proof is then very similar to the case $\Gamma_{T}=\emptyset$. Consequently, we only recall here the main steps for the sake of completeness.

1. First, for any $q \in X(\Omega)$, the residual form associated with (8) is defined by :

$$
r(q)=l(q)-a\left(A_{h}, q\right)
$$

Because of Lemma 1.1, setting $e_{A}:=A-A_{h}$, we obtain :

$$
r\left(e_{A}\right)=\left\|\frac{1}{\sqrt{\mu}} \operatorname{curl} e_{A}\right\|_{\Omega}^{2}
$$

Now, we define $\Psi \in H_{\Gamma_{N}}^{1}(\Omega)$ by :

$$
\int_{\Omega} \nabla \Psi \cdot \nabla \chi=\int_{\Omega} e_{A} \cdot \nabla \chi, \quad \text { for all } \chi \in H_{\Gamma_{N}}^{1}(\Omega) .
$$

Setting $\tilde{e}_{A}=e_{A}-\nabla \Psi$, we have $\tilde{e}_{A} \in X^{0}(\Omega) \subset X_{M}(\Omega)$. Using the Helmholtz decomposition (21), we have :

$$
e_{A}=w+\nabla(\varphi+\Psi)
$$

with $w \in H^{1}(\Omega)^{3} \cap X(\Omega)$ and $(\varphi+\Psi) \in H_{\Gamma_{N}}^{1}(\Omega)$. From (28) and using (4), similarly to the proof of Lemma 1.1 , we get

$$
r\left(e_{A}\right)=r(w)
$$

The point now is to bound from above the quantity $|r(w)|$ so that (27) occurs from (29) and (30). 
2. We introduce an operator $\mathcal{P}_{C l}$ defined by the identity (32) of [6] where for any edge $E$ belonging to $\overline{\Gamma_{N}}$, the face $F_{E}$ has to be chosen in $\Gamma_{N}$ :

$$
\begin{array}{cc}
\mathcal{P}_{C l}: H^{1}(\Omega)^{3} \cap X(\Omega) & \rightarrow X_{h}(\Omega), \\
v & \rightarrow \mathcal{P}_{C l} v .
\end{array}
$$

From lemma 5 of [6], we get :

$$
\left\{\begin{array}{c}
\sum_{T \in \mathscr{T}_{h}} \frac{1}{h_{T}^{2}}\left\|v-\mathcal{P}_{C l} v\right\|_{T}^{2} \leq C_{1}\|v\|_{1, \Omega}^{2}, \\
\sum_{F \in \mathcal{F}_{\text {int }} \cup\left(\mathcal{F} \cap \Gamma_{T}\right)} \frac{1}{h_{T}^{2}}\left\|v-\mathcal{P}_{C l} v\right\|_{F}^{2} \leq C_{2}\|v\|_{1, \Omega}^{2}
\end{array}\right.
$$

where the constants $C_{1}$ and $C_{2}$ do neither depend on $v$ nor on the mesh size $h$, but only on the shape regularity of the mesh. We use the fact that $r(w)=r\left(w-\mathcal{P}_{C l} w\right)$, so that, with classical Green formula, we get :

$$
\begin{aligned}
r(w) & =\sum_{T \in \mathscr{T}_{h}} \int_{T}\left(J_{s}-\operatorname{curl}\left(\frac{1}{\mu} \operatorname{curl} A_{h}\right)\right) \cdot\left(w-\mathcal{P}_{C l} w\right) \\
& -\sum_{F \in \mathcal{F}_{\text {int }}} \int_{F}\left[\frac{1}{\mu} \operatorname{curl} A_{h} \times n\right]_{F} \cdot\left(w-\mathcal{P}_{C l} w\right) \\
& -\sum_{F \in \mathcal{F} \cap \Gamma_{T}} \int_{F}\left(\frac{1}{\mu} \operatorname{curl} A_{h} \times n\right) \cdot\left(w-\mathcal{P}_{C l} w\right) .
\end{aligned}
$$

3. Finally, the use of Cauchy-Schwarz inequalities, discrete Cauchy-Schwarz inequalities, the properties (31) on $\mathcal{P}_{C l}$ and the stability relation (22) yield (27).

For completeness we now state the efficiency of the estimator (namely, the fact that the local estimator is bounded from above by the local error) :

Theorem 3.2 Let us define the patch of the element $T$ by $\mathscr{P}_{T}=\bigcup_{T^{\prime} \cap T \neq \emptyset} T^{\prime}$. Then, there exists a constant $C_{\text {down }}>0$, which does not depend on $A, A_{h}$ or $h$, such that:

$$
\eta_{T} \leq C_{\text {down }}\left\|\frac{1}{\sqrt{\mu}} \operatorname{curl}\left(A-A_{h}\right)\right\|_{\mathscr{P}_{T}} .
$$

Proof. The proof is here exactly the same as the one corresponding to the case $\Gamma_{T}=\emptyset$, since we do not need any Helmholtz decomposition, but only need to use classical bubble functions and inverse inequalities. It is available in previous papers (see for example [13]). 


\section{Numerical validation}

\subsection{Analytical solution}

Let us consider here the unit cube $\Omega=[0,1 / 2]^{3}$. We want to compute the magnetic flux density $B=\operatorname{curl} A$ given by (3) with the following data (see Figure 1) :

$$
\left\{\begin{array}{l}
J_{s}=\left(0,0,10^{7}\right)^{T} \\
\Gamma_{N}=\left\{(x, y, z) \in \partial \Omega \text { such that } x y z\left(z-\frac{1}{2}\right)=0\right\} \\
\Gamma_{T}=\partial \Omega \backslash \Gamma_{N} \\
\mu=4 \pi \cdot 10^{-7}
\end{array}\right.
$$

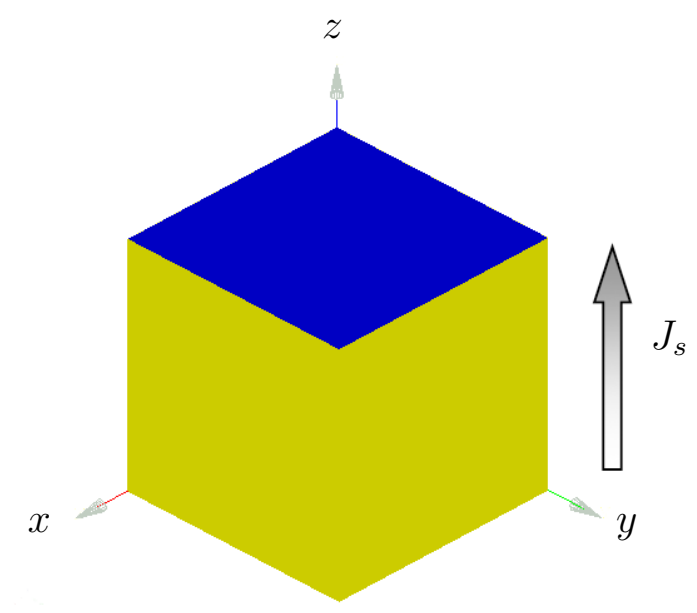

Figure 1: Domain configuration and eddy current $J_{s}$.

The solution of this problem is known analytically and is given by (see [2]) :

$$
\left\{\begin{array}{l}
B_{x}(x, y, z)=\frac{16 \cdot 10^{7} \mu}{\pi^{3}} \sum_{n=0}^{+\infty} \sum_{p=0}^{+\infty} \frac{\sin ((2 n+1) \pi x) \cos ((2 p+1) \pi y)}{(2 n+1)\left[(2 n+1)^{2}+(2 p+1)^{2}\right]} \\
B_{y}(x, y, z)=-\frac{16 \cdot 10^{7} \mu}{\pi^{3}} \sum_{n=0}^{+\infty} \sum_{p=0}^{+\infty} \frac{\cos ((2 n+1) \pi x) \sin ((2 p+1) \pi y)}{(2 p+1)\left[(2 n+1)^{2}+(2 p+1)^{2}\right]} \\
B_{z}(x, y, z)=0
\end{array}\right.
$$

Setting $B_{h}=\operatorname{curl} A_{h}$, the error $\left\|\frac{1}{\sqrt{\mu}}\left(B-B_{h}\right)\right\|_{\Omega}$ is plotted versus DoF in Figure 2(a) (where $D o F$ is the number of edges in the mesh), to show that the numerical approximated value of the magnetic flux density $B_{h}$ converges towards the exact one $B$ at order 1 in $h$ (or order $-1 / 3$ in $D_{o F}$ since $D_{o F}=O\left(1 / h^{3}\right)$ ), as theoretically expected by an ad hoc a priori convergence analysis (see for example [20]). The solution $B_{h}$ is moreover plotted in 
figure $2(\mathrm{~b})$ in the plane $z=1 / 2$ to show its qualitatively good behavior. Then, the ratio $\left\|\frac{1}{\sqrt{\mu}}\left(B-B_{h}\right)\right\|_{\Omega} / \eta$ is plotted versus $D o F$ in Figure 3 , in order to numerically confirm the reliability of the estimator given by $(27)$.

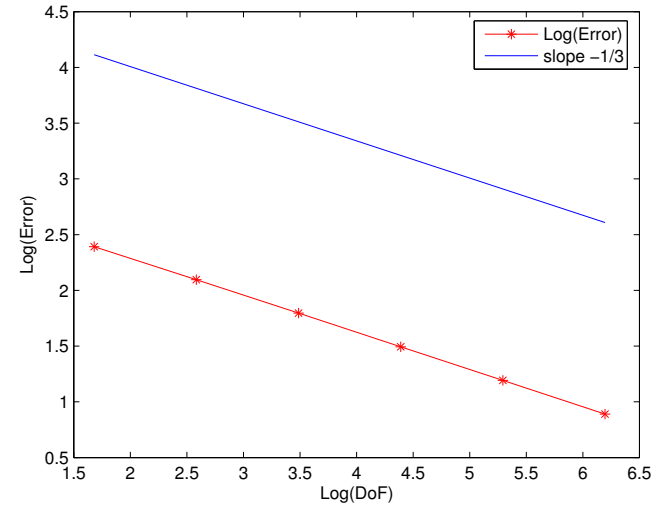

(a)

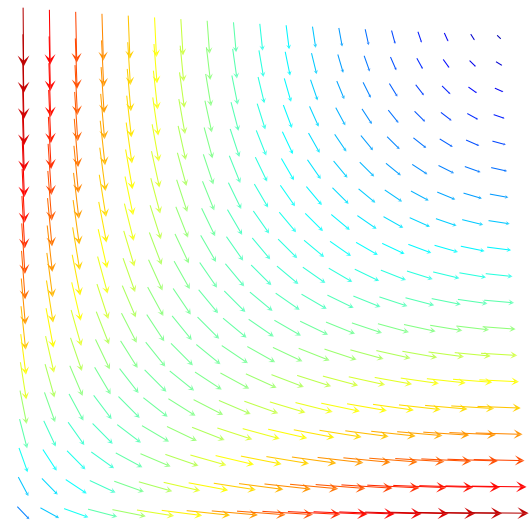

(b)

Figure 2: (a) : Convergence rate of the error, (b) : Approximated magnetic flux density field $B_{h}$ in the plane $z=1 / 2$.

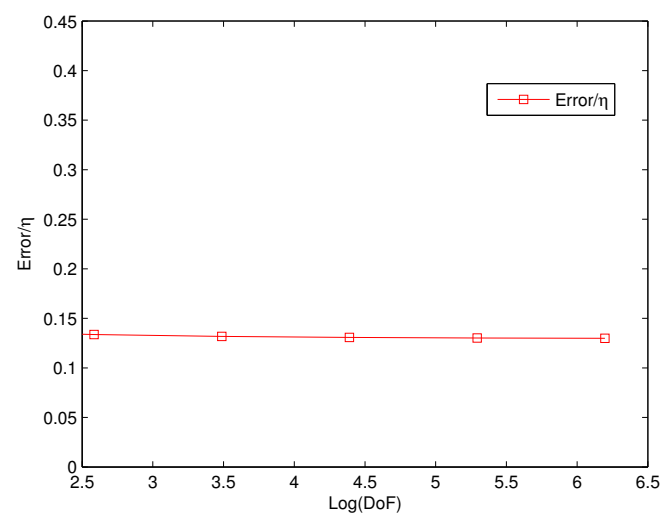

Figure 3: $\left\|\frac{1}{\sqrt{\mu}}\left(B-B_{h}\right)\right\|_{\Omega} / \eta$ versus $\log \left(D_{o} F\right)$.

\subsection{Singular solution}

We consider here a singular solution, in order to show that the estimator detects the elements where the error is the largest. The domain under consideration is defined by $\Omega=$ $L \times(0,1)$, where $L$ is the two dimensional L-shaped domain $L:=(-1,1)^{2} \backslash([0,1] \times[-1,0])$. 
The corresponding mesh is composed of 227155 tetrahedra. We want to compute the magnetic flux density $B=\operatorname{curl} A$ given by (3) with the following data (see Figure 4) :

$$
\left\{\begin{array}{l}
J_{s}=(0,0,1)^{T}, \\
\Gamma_{N}=\left\{(x, y, z)^{\top} \in \partial \Omega: z=0, \text { or } z=1, \text { or } y=0\right\} \\
\Gamma_{T}=\partial \Omega \backslash \Gamma_{N}, \\
\mu=1
\end{array}\right.
$$

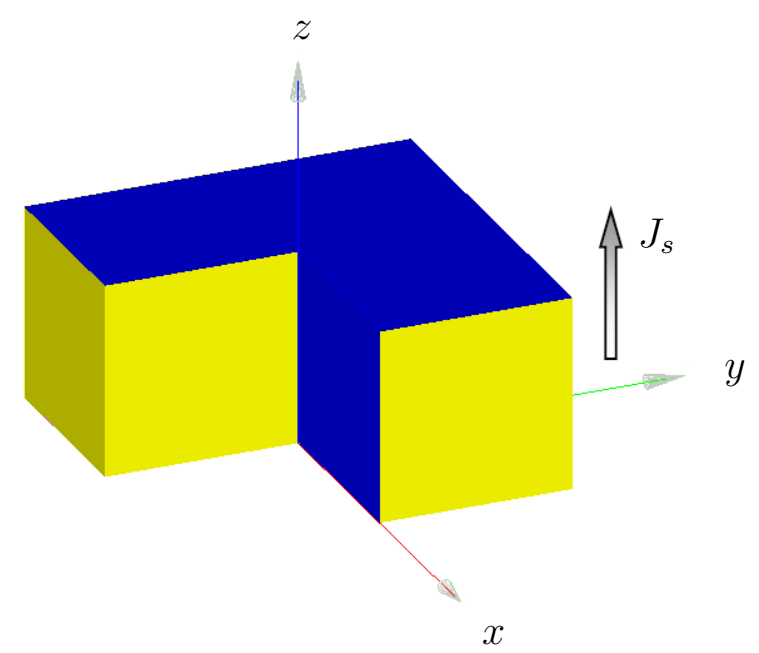

Figure 4: Domain configuration and eddy current $J_{s}$.

The exact solution is not known analytically but has an edge singularity along the edge $x=y=0$. Figure 5 shows that the estimator picks up correctly this singular behavior. Consequently, the efficiency is numerically confirmed (see Theorem 3.2) and a mesh-refinement strategy using this estimator could be successfuly used in order to adapt the mesh in the vicinity of the singularity.
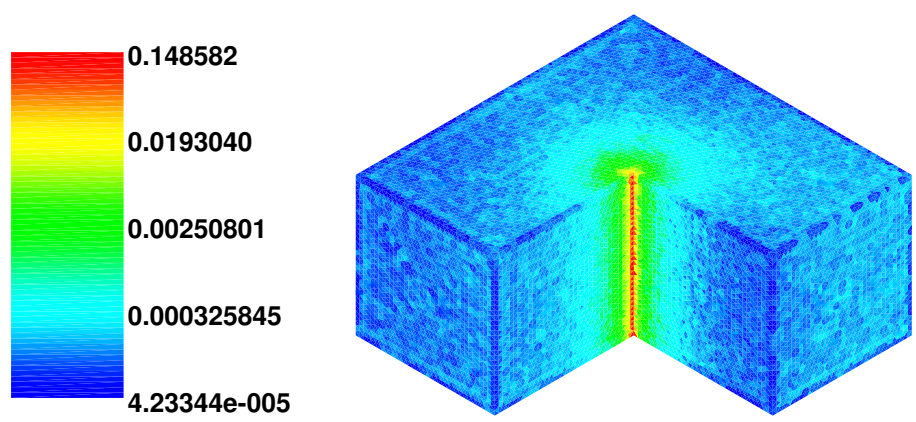

Figure 5: Local estimator map. 


\section{Conclusion}

In this paper, we were interested in the residual a posteriori error analysis of a magnetostatic problem given in its vectorial potential formulation in the context of a finite element approximation. Here, the model under consideration induces some mixed boundary conditions, what constitutes the originality of the present work. The key point needed in the proof of the reliability of this estimator is a new Helmholtz decomposition of vector fields, which has been established and constitutes the core of this contribution. Consequently, the reliability can be achieved using the same kind of proof as for the case of homogeneous boundary conditions. Numerical tests are then proposed, for a regular as well as for a singular solution, in order to illustrate the obtained theoretical results.

\section{References}

[1] M. Ainsworth and J.T. Oden. A posteriori error estimation in finite element analysis. Pure and Applied Mathematics (New York). Wiley-Interscience [John Wiley \& Sons], New York, 2000.

[2] R. Albanese and G. Rubinacci. Magnetostatic field computations in terms of twocomponent vector potentials. Int. J. Numer. Meth. Engng., 29:515-532, 1990.

[3] I. Babuška and W. C. Rheinboldt. Error estimates for adaptive finite element computations. SIAM J. Numer. Anal., 15(4):736-754, 1978.

[4] I. Babuška and W. C. Rheinboldt. A posteriori error estimates for the finite element method. Int. J. Numer. Meth. Engng., 12:1597-1615, 1978.

[5] I. Babuska and T. Strouboulis. The finite element method and its reliability. Numerical Mathematics and Scientific Computation. The Clarendon Press Oxford University Press, New York, 2001.

[6] R. Beck, R. Hiptmair, R.H.W. Hoppe, and B. Wohlmuth. Residual based a posteriori error estimators for eddy current computation. M2AN Math. Model. Numer. Anal., 34(1):159-182, 2000.

[7] M. Sh. Birman and M. Z. Solomyak. The Maxwell operator in domains with a nonsmooth boundary. Sibirsk. Mat. Zh., 28(1):i, 23-36, 1987.

[8] M.S. Birman and M.Z. Solomyak. $L_{2}$-theory of the Maxwell operator in arbitrary domains. Uspekhi Mat. Nauk, 42(6(258)):61-76, 247, 1987.

[9] J. Chen, Z. Chen, T. Cui, and L.B. Zhang. An adaptive finite element method for the eddy current model with circuit/field couplings. SIAM J. Sci. Comput., 32(2):10201042, 2010. 
[10] J. Chen, Y. Xu, and J. Zou. Convergence analysis of an adaptive edge element method for Maxwell's equations. Appl. Numer. Math., 59(12):2950-2969, 2009.

[11] S. Cochez-Dhondt and S. Nicaise. Robust a posteriori error estimation for the Maxwell equations. Comput. Methods Appl. Mech. Engrg., 196(25-28):2583-2595, 2007.

[12] M. Costabel, M. Dauge, and S. Nicaise. Singularities of Maxwell interface problems. M2AN Math. Model. Numer. Anal., 33(3):627-649, 1999.

[13] E. Creusé, S. Nicaise, Z. Tang, Y. Le Menach, N. Nemitz, and F. Piriou. Residualbased a posteriori estimators for the a/phi magnetodynamic harmonic formulation of the maxwell system. Mathematical Models and Methods in Applied Sciences, to appear.

[14] M. Dauge. Elliptic boundary value problems on corner domains, volume 1341 of Lecture Notes in Mathematics. Springer-Verlag, Berlin, 1988. Smoothness and asymptotics of solutions.

[15] V. Girault and P.A. Raviart. Finite element approximation of the Navier-Stokes equations, volume 749 of Lecture Notes in Mathematics. Springer-Verlag, Berlin, 1979.

[16] P. Grisvard. Elliptic problems in nonsmooth domains, volume 24 of Monographs and Studies in Mathematics. Pitman (Advanced Publishing Program), Boston, MA, 1985.

[17] R. H. W. Hoppe and J. Schöberl. Convergence of adaptive edge element methods for the 3D eddy currents equations. J. Comput. Math., 27(5):657-676, 2009.

[18] B. Janssen and G. Kanschat. Adaptive multilevel methods with local smoothing for $H^{1}$ - and $H^{\text {curl }}$-conforming high order finite element methods. SIAM J. Sci. Comput., 33(4):2095-2114, 2011.

[19] F. Kikuchi. On a discrete compactness property for the Nédélec finite elements. J. Fac. Sci. Univ. Tokyo Sect. IA Math., 36(3):479-490, 1989.

[20] P. Monk. Finite element methods for Maxwell's equations. Numerical Mathematics and Scientific Computation. Oxford University Press, New York, 2003.

[21] S. Nicaise and E. Creusé. A posteriori error estimation for the heterogeneous Maxwell equations on isotropic and anisotropic meshes. Calcolo, 40(4):249-271, 2003.

[22] M. Raffetto. Discrete compactness for edge elements in the presence of mixed boundary conditions. SIAM J. Numer. Anal., 42(5):1818-1829 (electronic), 2005.

[23] Z. Ren. Influence of the r.h.s. on the convergence behaviour of the curl-curl equation. IEEE Transactions on Magnetics, 32(3):655-658, 1996. 
[24] A.A. Rodriguez and M. Raffetto. Unique solvability for electromagnetic boundary value problems in the presence of partly lossy inhomogeneous anisotropic media and mixed boundary conditions. Math. Models Methods Appl. Sci., 13(4):597-611, 2003.

[25] J. Schöberl. A posteriori error estimates for Maxwell equations. Math. Comp., 77(262):633-649, 2008.

[26] R. Verfürth. A Review of a posteriori error estimation and adaptive mesh-refinement techniques. Wiley-Teubner, Chichester, Stuttgart, 1996. Smoothness and asymptotics of solutions.

[27] W. Zheng, Z. Chen, and L. Wang. An adaptive finite element method for the $H-\psi$ formulation of time-dependent eddy current problems. Numer. Math., 103(4):667-689, 2006.

[28] W. Zheng and F. Zhang. Adaptive finite element frequency domain method for eddy current problems. Comput. Methods Appl. Mech. Engrg., 197(13-16):1233-1241, 2008. 